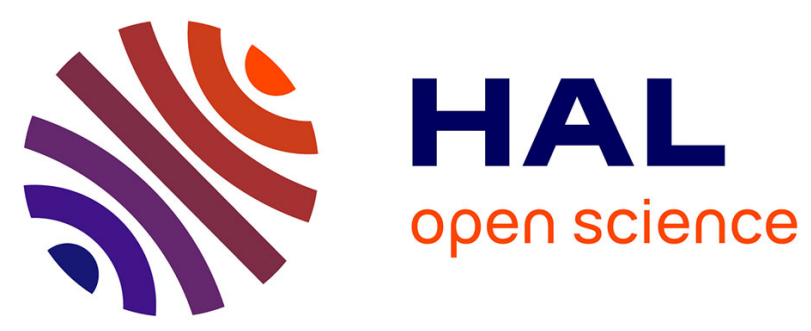

\title{
The Iterative 2D Windowed Fourier Transform: A SOS Approach to Speckle Noise Reduction in Digital Holography
}

\author{
Silvio Montrésor, Pascal Picart, Mayssa Karray
}

\section{To cite this version:}

Silvio Montrésor, Pascal Picart, Mayssa Karray. The Iterative 2D Windowed Fourier Transform: A SOS Approach to Speckle Noise Reduction in Digital Holography. 2017 25th European Signal Processing Conference (EUSIPCO), Aug 2017, Kos, Greece. pp.1544-1548, 10.23919/EUSIPCO.2017.8081468 . hal-02516974

\section{HAL Id: hal-02516974 \\ https://hal.science/hal-02516974}

Submitted on 24 Mar 2020

HAL is a multi-disciplinary open access archive for the deposit and dissemination of scientific research documents, whether they are published or not. The documents may come from teaching and research institutions in France or abroad, or from public or private research centers.
L'archive ouverte pluridisciplinaire HAL, est destinée au dépôt et à la diffusion de documents scientifiques de niveau recherche, publiés ou non, émanant des établissements d'enseignement et de recherche français ou étrangers, des laboratoires publics ou privés. 


\section{The Iterative 2D Windowed Fourier Transform: A SOS Approach to Speckle Noise Reduction in Digital Holography}

\author{
Silvio Montrésor \\ LAUM, UMR CNRS 6613, \\ Université du Maine, France \\ Email: silvio.montresor@univ-lemans.fr
}

\author{
Pascal Picart \\ LAUM, UMR CNRS 6613, \\ Université du Maine, France \\ Email: pascal.picart@univ-lemans.fr
}

\author{
Mayssa Karray \\ Unité de l'état solide, \\ 3018 Sfax, Tunisie, \\ Email: mayssakarray@gmail.com
}

\begin{abstract}
This paper addresses the problem of the reduction of speckle noise corrupting phase images from laser digital holography technique. It present an iterative denoising algorithm based on the 2-D windowed Fourier Transform. The algorithm is a new approach based on the SOS procedure recently proposed by $Y$. Romano and M. Elad adapted to phase processing. The approach is proposed in the case of the 2-D windowed Fourier Transform algorithm applied to phase maps which constitutes the state of the art in the field of digital holography. The reason is that the sum and difference operations used in the SOS procedure cannot be computed directly on phase maps but using a sine and cosine representation in order to avoid $2 \pi$ phase jumps. Results on simulated phase maps including realistic noise conditions encountered in digital holography show the advantage of the proposed iterative approach. The paper proposes the application of the method to denoising of phase images from digital three color holography applied to cracks characterization of a composite materiel under mechanical strength test.
\end{abstract}

Keywords-denoising, image processing, speckle noise, phase, iterative algorithms, windowed Fourier Transform, digital holography

\section{INTRODUCTION}

The digital holography technique is a laser based optical process. It allows measurements of displacement fields and surface shape. Due to contactless measurements, rough surfaces characterization of objects can be obtained with very good accuracy.

Digital holography technique is based on a coherent mixing of a reference wave and an object wave which results from light diffraction of any object supposed having opaque and rough surface. An image sensor (CMOS or CCD) records fringe patterns delivered by the coherent mixing. Figure 1 exhibits the digital holography principle. If the hologram is recorded [1] in accordance with the Shannon conditions, we obtain an image of the object wave-front from digital reconstruction of the hologram. The complex field of the object is computed by Single Fast Fourier Transform (S-FFT) or Double Fast Fourier Transform (D-FFT) algorithms using adjustable magnification [2]. The numerical calculation of the diffracted field yields a complex amplitude $O_{0}(x, y)$ sampled on a grid corresponding to the number of reconstruction points of the algorithm. The amplitude image (modulus), $A_{r}$, and phase image, $\psi_{r}$ (argument of the field) are derived from the complex amplitude, namely:

$$
\begin{gathered}
A_{r}(x, y)=\left|O_{0}(x, y)\right| \\
\psi_{r}(x, y)=\operatorname{atan} 2\left\{\frac{\Im m\left[O_{0}(x, y)\right]}{\Re e\left[O_{0}(x, y)\right]}\right\} \bmod (2 \pi) .
\end{gathered}
$$

Consequently to computation by the means of the arctangent function, $\psi_{r}$ is wrapped in the interval $[-\pi,+\pi]$, modulo $2 \pi$. In most cases the randomness of the phase is due to the roughness of the object. The reconstructed object is then subject to the phenomenon of speckle noise. Phase estimation of the reconstructed optical field is the key for a large number of applications of digital holography. In our laboratory, we focus especially on the study of acoustic phenomena using a technique of ultra-fast digital holography with a sampling rate up to $100 \mathrm{kHz}$ [3]. The reconstructed amplitude is amplitude modulated by a speckle pattern due to the coherent light and consequently, speckle acts as a multiplicative noise. Metrology applications require only optical phase so we focus on phase changes over time. The quantity of interest is a phase difference between two instants, allowing to follow-up the evolution of a phenomenon over time. Taking into account Doppler effect, the phase difference is proportional to the displacement field of the object between the two instants. As the optical phase is calculated from the arctangent function, then it is wrapped. Phase must be unwrapped in order to access physical kinematic quantities on the object [4].

\section{SPECKLE NOISE IN DOPPLER PHASE}

Usually, a speckle decorrelation phenomenon appears [5] when the object is distorted under the effect of any applied constraint, which may be mechanical, acoustic, thermal, etc... It adds a high spatial frequency noise to the useful signal. Spatial correlation width of this noise is related to the size of the observable speckle grain in the amplitude image. Consequently, the phase image needs a filtering process in order to be correctly exploited for a confrontation with a physical model of the studied object. The speckle decorrelation 


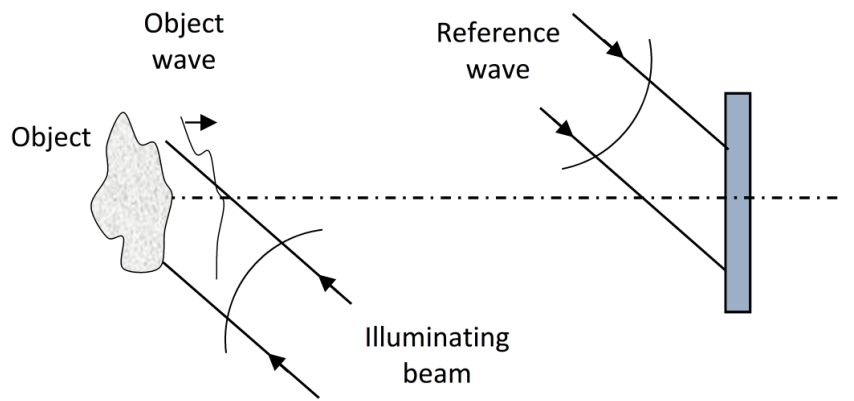

Fig. 1. Holography principle.

has been studied by some authors [6]-[9]. Decorrelation can be highlighted from differences of two phases measured at two different instants. The second order probability density function of the phase [10] is of interest. The computation of the joint probability density function of two speckle phases $\psi_{r 1}$ and $\psi_{r 2}$ has been given in [11], [12]. Given $\varepsilon$ the noise induced by speckle decorrelation between two extracted phases at two different instants and $\Delta \varphi$ the phases variation due to structure modification, then it comes $\psi_{r 2}=\psi_{r 1}+\varepsilon+\Delta \varphi$. $\Delta \varphi$ is considered as a deterministic variable. The probability density function of $\varepsilon$ depends on the modulus of the complex coherence factor between two speckle fields (noted $\mu$ ). Considering $\beta=|\mu| \cos (\varepsilon)$, the probability density function of phase noise $\varepsilon$ is given by:

$p(\beta)=\frac{1-|\mu|^{2}}{2 \pi}\left(1-\beta^{2}\right)^{-3 / 2}\left(\beta \arcsin \beta+\frac{\pi \beta}{2}+\sqrt{\left(1-\beta^{2}\right)}\right)$.

Equation 3 describes the probability of noise $\varepsilon$ in the difference between two instants. Generally, to preserve $2 \pi$ phase jumps in the wrapped phase map, the processing is applied on sine and cosine of the phase variations.

\section{THE 2-D WINDOWED FOURIER TRANSFORM}

The 2-D windowed Fourier transform filter (WFT2F) method is based on a local Fourier transform (FT) which can take into account the non-stationary characteristic of the speckle noise [13], [14]. So, frequency components of a fringe pattern could be extracted from noise with more accurate efficiency than with a Fourier transform (FT) computed overall phase maps as acts for example the Wiener filter method. In a same way as wavelet transforms but adapted to a complex representation, the filtering process consists in applying a threshold on the modulus of the 2-D Fourier coefficients, letting phase unchanged. Finally, an inverse 2-D windowed Fourier transform (IWFT2F) of the filtered frequency domain signal is computed to get the modulo $2 \pi$ phase of the fringe pattern. The WFT2F can be seen as a projection of phase map onto 2-D oscillatory functions $G_{\mu, \nu, \xi, \eta}(x, y)$ which are localized both in the spatial and the frequency domain. Let $s(x, y)$ the input phase map, it follows:

$$
W F T_{s}(\mu, \nu, \xi, \eta)=\iint s(x, y) G_{\mu, \nu, \xi, \eta}^{*}(x, y) d x d y .
$$

In the above equation, the symbol $*$ denotes the complex conjugate operation. $W F T_{s}$ denotes the WFT2F applied to image $s$. $G$ functions constitutes the atoms of the transformation and are obtained with translations and modulation in the spatial domain of a window function $g(x, y)$ :

$$
G_{\mu, \nu, \xi, \eta}(x, y)=g(x-\mu, y-\nu) \exp (j(\xi x+\eta y)),
$$

which is a normalized Gaussian function given as:

$$
g(x, y)=\frac{1}{\sqrt{\pi \sigma_{x} \sigma_{y}}} \exp \left(-\left(\frac{x^{2}}{2 \sigma_{x}^{2}}+\frac{y^{2}}{2 \sigma_{y}^{2}}\right)\right) .
$$

Variables $\sigma_{x}$ and $\sigma_{y}$ are standard deviations of the Gaussian function in $x$ and $y$ directions. The filtering process is achieved with a hard thresholding applied onto WFT2F coefficients. Denote $W=W F T_{s}(\mu, \nu, \xi, \eta)$, the transformation coefficients and $\bar{W}$ the thresholded coefficients we get:

$$
\bar{W}= \begin{cases}W & \text { if }|W| \geq T \\ W=0 & \text { if }|W|<T\end{cases}
$$

$T$ is the threshold which value is set in order to suppress noise components. The overbar of WFT2F coefficients denotes the filtering process which modifies input coefficients. Finally an IWFT2F is applied on filtered coefficients $\bar{W}$ in order to get the denoised image,

$$
d(x, y)=\iiint \int \bar{W} G_{\mu, \nu, \xi, \eta}(x, y) d \mu d \nu d \xi d \eta .
$$

The phase $\phi(x, y)$ can be extracted from the output image $d(x, y)$ with:

$$
\phi(x, y)=\operatorname{atan} 2\left\{\frac{\Im m[d(x, y)]}{\Re e[d(x, y)]}\right\} \bmod (2 \pi) .
$$

The discrete version of this algorithm leads to the setting of an amount of eight parameters which define the window size, the threshold value and six parameters for discretization of the frequency axis. For the evaluation, it has been kept values proposed by the author [13] which seem to be well adapted to the processing of fringe patterns.

\section{THE SOS PROCEDURE}

The SOS procedure recently proposed [15] is a generic recursive function that processes denoising method as a blackbox and has the ability to push it forward to improve its performance. The SOS procedure allows to improve performances of some families of denoising algorithms like NLmeans [16], K-SVD [17] or BM3D [18]. Unlike the above methods, instead of adding the residual which mostly contains noise [19] back to the noisy image, or filtering the previous estimate over and over again which could lead to oversmoothing, the authors suggest strengthening the signal by leveraging on the availability of the denoised image. More precisely, given an initial estimation of the cleaned image, improved results can be achieved by iteratively repeating the following SOS procedure:

- Strengthen the signal by adding the previous denoised image to the noisy input image ;

- Operate the denoising on the strengthened image ; 
- Subtract the previous denoised image from the restored signal-strengthened outcome.

The equation that describes this procedure is written in the following form:

$$
x^{k+1}=F\left(y+x^{k}\right)-x^{k},
$$

with $x^{0}=0$. In the above equation $y$ is the initial observation (noisy image), $x^{k}$ terms represent the iterated estimations of the noisy free image and $F$ is an operator representing a denoising algorithm. A performance improvement is then achieved since the signal-strengthened image can be denoised more effectively compared to the noisy input image, due to the improved signal-to-noise ratio (SNR). The underlying hypothesis on the observation is the classical additive noise model, with the statistical independence between the clean image and the noise. In [15], the authors introduce a $\rho$ parameter in order to control signal emphasis, so iterated signal estimates become:

$$
x^{k+1}=F\left(y+\rho x^{k}\right)-\rho x^{k} .
$$

This control parameter improves the convergence property of the SOS procedure.

\section{A. Adaptation to WFT2F algorithm}

In order to use WFT2F algorithm as a black box of the SOS procedure, some precautions have to be taken to avoid artificial phase jumps at $-\pi$ and $+\pi$ angles that could arise in the summation operation needed to compute strengthened signal, and in the difference operation which compute the final iterate estimate. First of all, output phase map resulting from WFT2F algorithm is transformed in cosine and sine components. Then an arithmetic weighted mean is computed on cosine and sine components in order to get strengthened components, resulting phase map is obtained from angle computed with strengthened components:

$$
\phi_{\text {strength }}=\operatorname{atan} 2\left\{\frac{\frac{1}{1+\rho}\left(\cos \left(\phi_{y}\right)+\rho \cos \left(\phi_{k}\right)\right)}{\frac{1}{1+\rho}\left(\sin \left(\phi_{y}\right)+\rho \sin \left(\phi_{k}\right)\right)}\right\} \bmod (2 \pi) .
$$

Same procedure with different weightings is used in order to compute difference between denoised strengthened phase map $\phi_{\text {strength }}$ and the $k^{\text {th }}$ iterate estimate of the noise free phase map $\phi_{y}$.

\section{Database and evaluation}

The database for the benchmark consists of simulated fringe images in which the type of the fringe pattern and the noise level are controlled while statistics follow 3 . As the database and the principle of simulation have been published in previous articles [19], [20], only its main characteristics are recalled here. The use of several fringe pattern permits to consider a certain "fringe diversity" so as to establish statistics on the obtained results. For this purpose, five different fringe patterns are chosen. In addition, five values for the SNR were simulated by decreasing the SNR value with adding to the simulation a wavelength change. It leads to 25 noisy phase maps with cosine SNR varying from about $3 \mathrm{~dB}$ to $12 \mathrm{~dB}$. This helps to simulate degraded phase images according to real experimental conditions for which a lot of limiting factors may influence the quality of the measurement. The metrics used for this evaluation are the phase error $\sigma_{\phi}$ which represents the standard deviation of the wrapped phase difference beetween the denoised and the noisy phase maps [20], and the gain of SNR between noisy and denoised image. The choice of phase error metrics is justified by metrological applications underlying with digital holography technique. As the processed phase maps are about zero mean, this metric is closely link with the PSNR mostly used in the evaluation algorithms in the image processing community. In order to compare our new approach with other denoising methods, algorithms that performances have been previously published [20] have been selected. Selected methods are: WFT2F, discrete wavelets as Daubechies wavelets and symlets (Daub4, Daub8, sym4, sym8) [21], curvelets [22], median filters, BM3D [18], NLmeans [16] and a sparse approach (Spadedh) [23].

\section{RESULTS AND DISCUSSION}

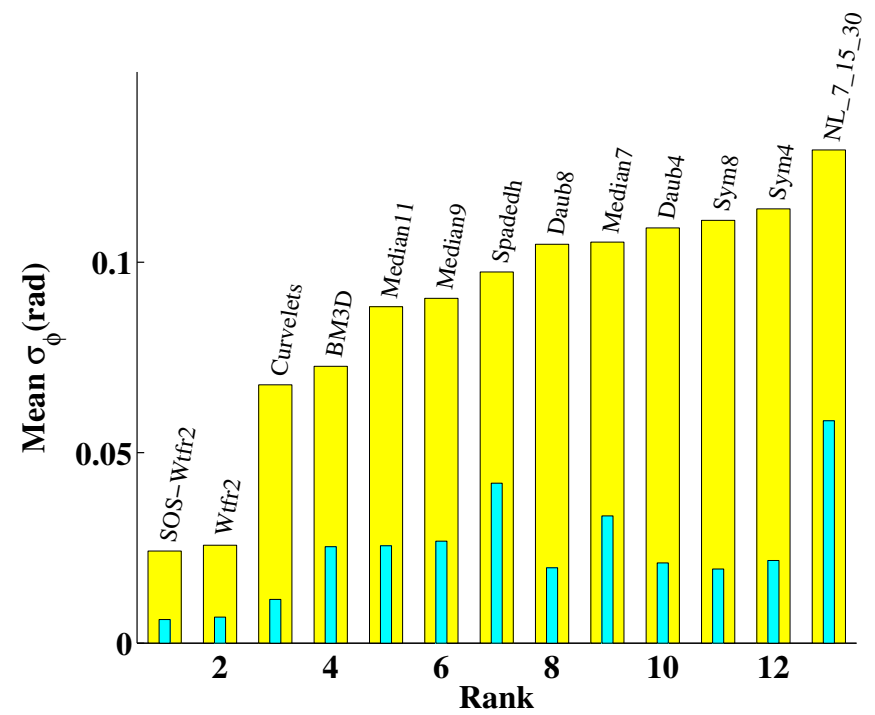

Fig. 2. Ranking obtained for the average value of $\sigma_{\phi}$. Within each yellow bar, a green bar corresponds to the standard deviation associated with the values displayed for the entire database.

Figure 2 exhibits the ranking of denoising algorithms together with the phase error. The SOS-WFT2F algorithm (denoted SOS-Wtfr2 in the figures) appears to be the best one. It can also clearly see that it presents very close results compared to WFT2F (denoted Wtfr2 in the figures). Looking to results on the five lowest input SNR images (see Tab.1) of the database reveals that SOS procedure performs better when the input SNR is weak. So SOS-WFT2F has to be applied when acquisition conditions are severe. Concerning SOS algorithm parameters, we can notice that the best values of phase errors have been found for few iterations (2 to 4$)$ and $\rho=1.4$ for signal emphasis parameter. 


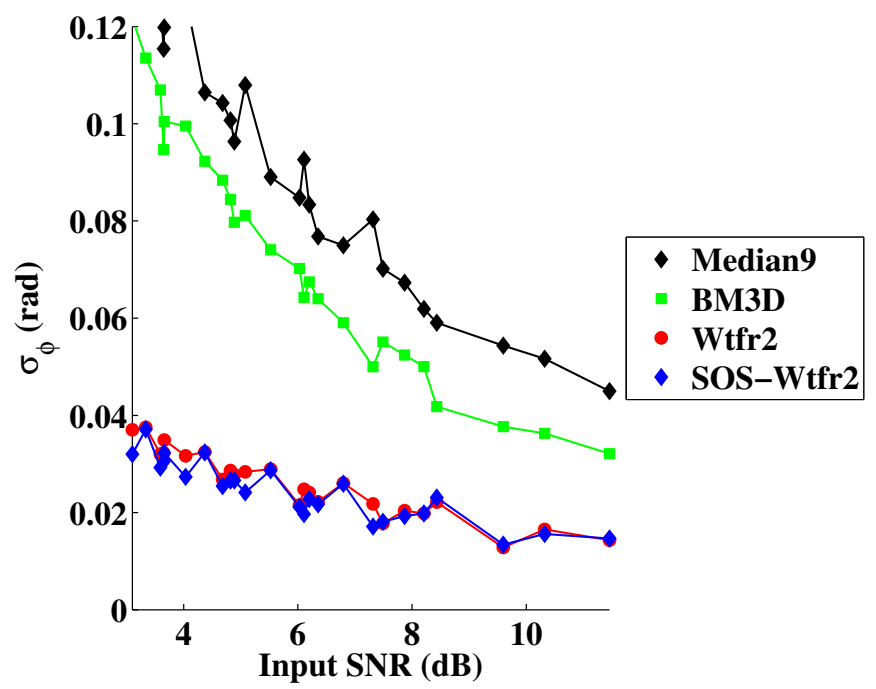

Fig. 3. Evolution of the average value of $\sigma_{\phi}$ according to the input SNR.

TABLE I

RESULTS OF WTFR2 AND SOS-WTFR2 METHODS WITH $\sigma_{\phi}$ AND $O_{S N R}$ (OUTPUT SNR) METRICS. $I_{S N R}$ IS THE INPUT SNR.

\begin{tabular}{|c|c|c|c|c|}
\hline Method: & Wtfr2 & SOS-Wtfr2 & Wtfr2 & SOS-Wtfr2 \\
\hline \hline$I_{S N R}(\mathrm{~dB})$ & $\sigma_{\phi}$ (rad.) & $\sigma_{\phi}($ rad. $)$ & $O_{S N R}(\mathrm{~dB})$ & $O_{S N R}(\mathrm{~dB})$ \\
\hline \hline 3.10 & 0.0370 & 0.0319 & 28.62 & 29.91 \\
3.33 & 0.0375 & 0.0371 & 28.56 & 28.66 \\
3.59 & 0.0320 & 0.0292 & 30.11 & 30.89 \\
3.65 & 0.0322 & 0.0304 & 29.57 & 30.10 \\
3.66 & 0.0349 & 0.0323 & 31.05 & 31.68 \\
\hline
\end{tabular}

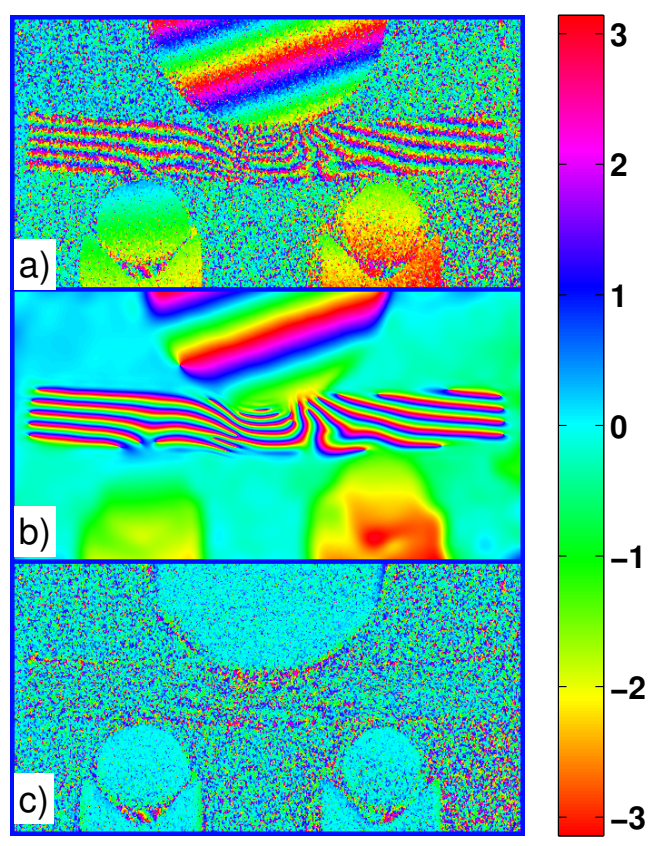

Fig. 4. Application to phase map denoising. (a) Noisy phase extract from blue laser beam, (b) denoised phase, (c) noise extracted by the algorithm.

\section{AppliCATiON}

The SOS-WFT2F algorithm has been applied to threecolor holographic interferometry for accurate displacement measurement. The goal is to achieve detection of precursor crack in composite materials submitted to a strength test [24], [25]. The simultaneous recording of three laser wavelengths using a triple CCD sensor allows the characterization of shear strains at the lateral surface of the sample. It provides a pertinent parameter to detect precursor crack in the structure, long before it becomes visible on the real time stress/strain curve. Figure 4 shows a phase map obtained with the set-up that was processed using SOS-WFT2F for the blue laser. The SOS-WFT2F algorithm allows us to obtain denoised phase maps which will be used for the detection of crack in an efficient manner. This last point will be detailed in a next paper. Time computation of SOS-WFT2F is about $5 \mathrm{~min}$ for an 1024x1024 pixels image on a laptop core-5 pc.

\section{CONCLUSION}

This paper presents a new iterative algorithm based on the 2-D windowed Fourier Transform in combinaison with the SOS procedure. In order to avoid smoothing of $2 \pi$ phase jumps, a sine and cosine representation is proposed for the sum and difference operations of the SOS procedure. Results on simulated phase maps including realistic noise conditions encountered in digital holography show the superiority of the proposed approach compared to a set of denoising algorithms. In addition, this approach yields performances well beyond the state of the art constituted by the 2-D windowed Fourier Transform algorithm. The application or this new method to denoising of phase images demonstrates it applicability to digital three color holography for characterization of composite materials.

\section{ACKNOWLEDGMENT}

This research is funded from the French National Agency for Research (ANR) under grant agreement number ANR- 14ASTR-0005-02.

\section{REFERENCES}

[1] P. Picart and J. Leval, "General theoretical formulation of image formation in digital fresnel holography," J. Opt. Soc. Am. A, vol. 25, pp. 1744-1761, 2008.

[2] P. Picart and J. Li, Digital holography. London: ISTE-Wiley, 2012.

[3] J. Poittevin, F. Gautier, C. Pezerat, and P. Picart, "High-speed holographic metrology: principle, limitations, and application to vibroacoustics of structures," Optical Engineering, vol. 55, no. 12, pp. $121717-$ $121729,2016$.

[4] H. Xia, S. Montresor, R. Guo, J. Li, F. Yan, H. Cheng, and P. Picart, "Phase calibration unwrapping algorithm for phase data corrupted by strong decorrelation speckle noise," Optics Express, vol. 24, no. 25, pp. 28713-28 730, 2016

[5] P. Owner-Petersen, "Decorrelation and fringe visibility: on the limiting behavior of various electronic speckle-pattern correlation interferometers," J. Opt. Soc. Am.A, vol. 8, pp. 1082-1089, 2008.

[6] M. Lehmann, "Decorrelation-induced phase errors in phase shifting speckle interferometry," Appl. Opt., vol. 36, pp. 3657-3667, 1997.

[7] — "Phase-shifting speckle interferometry with unresolved speckles: A theoretical investigation," Opt. Comm., vol. 128, pp. 325-340, 1996.

[8] — - "Optimization of wave-field intensities in phase-shifting speckle interferometry," Opt. Comm., vol. 118, pp. 199-206, 1995.

[9] P. Picart, J. Leval, D. Mounier, and S. Gougeon, "Time-averaged digital holography," Optics. Letters., vol. 28, pp. 1900-1902, 2003.

[10] J. C. Dainty, A. E. Ennos, M. Franon, J. Goodman, T. S. McKechnie, and G. Parry, Laser Speckle and Related Phenomena. Berlin: ISTE-Wiley, 1975. 
[11] D. Middleton, Introduction to Statistical Communication Theory. New York: Mc Graw Hill, 1960

[12] W. B. Davenport and W. L. Root, Random Signals and Noise. New York: Mc Graw Hill, 1958.

[13] Q. Kemao, "Two-dimensional windowed fourier transform for fringe pattern analysis: Principles, applications and implementations," Opt. \& Las. Eng., vol. 45, no. 12, pp. 1146-1192, 2007.

[14] Q. Kemao, L. Nam, L. Feng, and S. Soon, "Comparative analysis on some filters for wrapped phase maps," Appl. Opt., vol. 46, no. 12, pp. 7412-7418, 2007.

[15] Y. Romano and M. Elad, "Boosting of image denoising algorithms," SIAM J. Imaging Sciences, vol. 8, no. 2, pp. 1187-1219, 2015.

[16] A. Buades, B. Coll, and J. Morel, "A review of image denoising algorithms, with a new one," Multiscale Modeling and Simulation, vol. 45 , no. 5 , pp. $490-530,2005$.

[17] M. Elad and M. Aharon, "Image denoising via sparse and redundant representations over learned dictionaries," IEEE Trans. Image Process., vol. 15, pp. 3736-3745, 2006.

[18] V. K. K. Dabov, A. Foi and K. Egiazarian, "Image denoising by sparse 3d transform-domain collaborative filtering," IEEE Trans. Image Process. vol. 16, no. 8, pp. 2080-2095, 2007.

[19] S. Montresor, P. Picart, O. Sakharuk, and L. Muravsky, "Error analysis for noise reduction in 3D deformation measurement with digital color holography," Journal of the Optical Society of America B, vol. 34, no. 5, pp. B9-B15, 2017.

[20] S. Montresor and P. Picart, "Quantitative appraisal for noise reduction in digital holographic phase imaging," Optics Express, vol. 24, no. 13, pp. 14 322-14343, 2016.

[21] S. G. Mallat, A wavelet tour of signal processing. San Diego: Academic Press, 1999.

[22] J. Starck, E. J. Candes, and D. L. Donoho, "The curvelet transform for image denoising," IEEE Trans. Image Process., vol. 11, no. 6, pp. 670-684, June 2002.

[23] P. Memmolo, I. Esnaola, A. Finizio, M. Paturzo, P. Ferraro, and A. M. Tulino, "Spadedh: a sparsity-based denoising method of digital holograms without knowing the noise statistics," Opt. Express, vol. 20, no. 15, pp. 17250-17 257, 2012.

[24] M. Karray, P. Slangen, and P. Picart, "Comparison between digital fresnel holography and digital image-plane holography: The role of the imaging aperture," Exp. Mechanics, vol. 52, pp. 1275-1290, 2012.

[25] M. Karray, C. Poilane, M. Gargouri, and P. Picart, "Evaluation of interlaminar shear of laminate by $3 \mathrm{~d}$ digital holography," Opt. \& Las. Eng., vol. 92, pp. 57-62, 2017. 